(C) 1982. The Genetical Society of Great Britain

\title{
A MEASUREMENT OF DISPERSAL IN THE GRASSHOPPER PODISMA PEDESTRIS (ORTHOPTERA: ACRIDIDAE)
}

\author{
N. H. BARTON and G. M. HEWITT \\ Department of Genetics, University of Cambridge, Cambridge CB2 3EH, U.K.; \\ School of Biological Sciences, University of East Anglia, Norwich NR4 7TJ, U.K.
}

Received 7.x.81

\section{SUMMARY}

The dispersal rate of the grasshopper Podisma pedestris has been measured, with the aim of interpreting the width of a chromosomal cline. 171 adults were marked individually, and released within the cline. 169 movements were seen over three subsequent scorings; the distribution of distances, after correction for the loss of long distance dispersants, was close to a normal curve, but there was an initial shift of ten metres, perhaps towards a better habitat. The linear variance increased at about $21.4 \mathrm{~m}^{2}$ day $^{-1}$, which corresponds to a standard deviation of $20.7 \mathrm{~m} \mathrm{gen}^{-1 / 2}$ over a 20 day life span. Statistical uncertainty in this estimate can be expressed using a distribution-free maximum likelihood method, which gives support limits of $18 \cdot 6-27.0 \mathrm{~m}^{-1 / 2}$ gen $^{-1 / 2}$. However, the main errors come from extrapolating from this experiment to the cline as a whole.

\section{INTRODUCTION}

DISPERSAL has been studied for several reasons-to help control the spread of pests, to understand a feature of behaviour important for population dynamics, and to find out how the movement of genes affects evolution. This paper is only concerned with the last motive; we began to look at dispersal whilst studying a cline between two chromosomal races of the grasshopper Podisma pedestris (Barton and Hewitt, 1981a). The rate of gene flow is a crucial parameter in interpreting spatial patterns. In particular, the width of a cline depends on the ratio between dispersal and selection (Haldane, 1948), and so by finding the first of these, we can deduce the strength of the selection maintaining the cline.

When selection is weak, the genetic effects of dispersal depend only on the mean and variance of parent-offspring distance (Nagylaki, 1975). The shape of the distribution of movements will therefore only be of interest in so far as it affects these two parameters; we will describe a mark-releaserecapture experiment designed to measure the dispersal rate within a section of cline whose width was known accurately.

\section{METHODS}

\section{(i) Life history}

Podisma pedestris is a large, flightless grasshopper with a wide distribution in the European Alps, Appennines, Pyrenees and Siberia. In the region studied, near Col de Tende in the Alpes Maritimes, it is found in a variety of habitats, the only strict requirements being for open, sunny sites and bare patches of earth in which to lay eggs. This catholic taste means that 
Podisma is abundant, and more or less continuously distributed above $1500 \mathrm{~m}$. The life cycle is annual; the eggs hatch as soon as the snow melts, and the cryptically coloured nymphs take about four weeks to develop into adults (longer in bad weather). Adult females start to lay eggs a week after emerging, and continue laying until the snow comes again.

\section{(ii) Experimental site}

The site of the mark-release-recapture experiment had to be within the cline, open and accessible, and densely populated. Unfortunately, these conditions are hard to reconcile; the cline runs along a region of generally low abundance, and steep slopes. The most suitable site, a fairly flat area of short grass and gravel, still had many nymphs when the experiment began, and these would have lost any marks when they moulted. Grasshoppers were therefore collected from a dense but precipitous site $200 \mathrm{~m}$ away, at the same point in the cline. Their subsequent dispersal could therefore be partly due to crowding, or to a search for a better area; however, the density of the released insects was much less than at their home area, and about the same as the native adult density in the release site.

\section{(iii) Marking and release}

Grasshoppers were marked individually on the evening of their capture by applying oil paint to their thorax, in a four spot, five colour code. The next morning 80 females and 91 males were released from three points (fig. 1), whilst they were still cold and immobile. Podisma will survive for a week or more in collecting tins with low mortality, and the insects showed no sign of agitation after their release. As well as these three releases, 132 insects were released from peripheral sites, in an attempt to measure long-distance movements. Only two of these were picked up later, and so these data have not been analysed in detail.

\section{(iv) Scoring}

The shape of the searched area is important in determining the efficiency of a mark-release-recapture experiment. For a given amount of effort, a fixed area can be searched, but the shape of this area determines the chance of detecting long-distance migrants. The need to measure movements over large distances, which contribute more to the rate of gene flow, must be balanced against the need to detect a reasonable proportion of the insects released. Accordingly, we used a long strip, $20 \mathrm{~m} \times 10 \mathrm{~m}$, bent in the middle to avoid steep slopes. Although any grasshoppers which moved more than $10 \mathrm{~m}$ might have been lost, there was still a reasonable chance of detecting movements of up to $70 \mathrm{~m}$ or so (fig. 1).

This strip was divided into three areas, each of which was scored by a pair of independent scorers on three occasions, three, six and eight days after the release. On each day the weather was fine, and did not change during the two hour scoring period. Over the three days, each person scored one area twice, and then moved on to a new area; no pair remained together for more than one day. This allowed the effects of scoring ability and of areas to be disentangled, and meant that any differences in ability between the scorers were averaged out. 


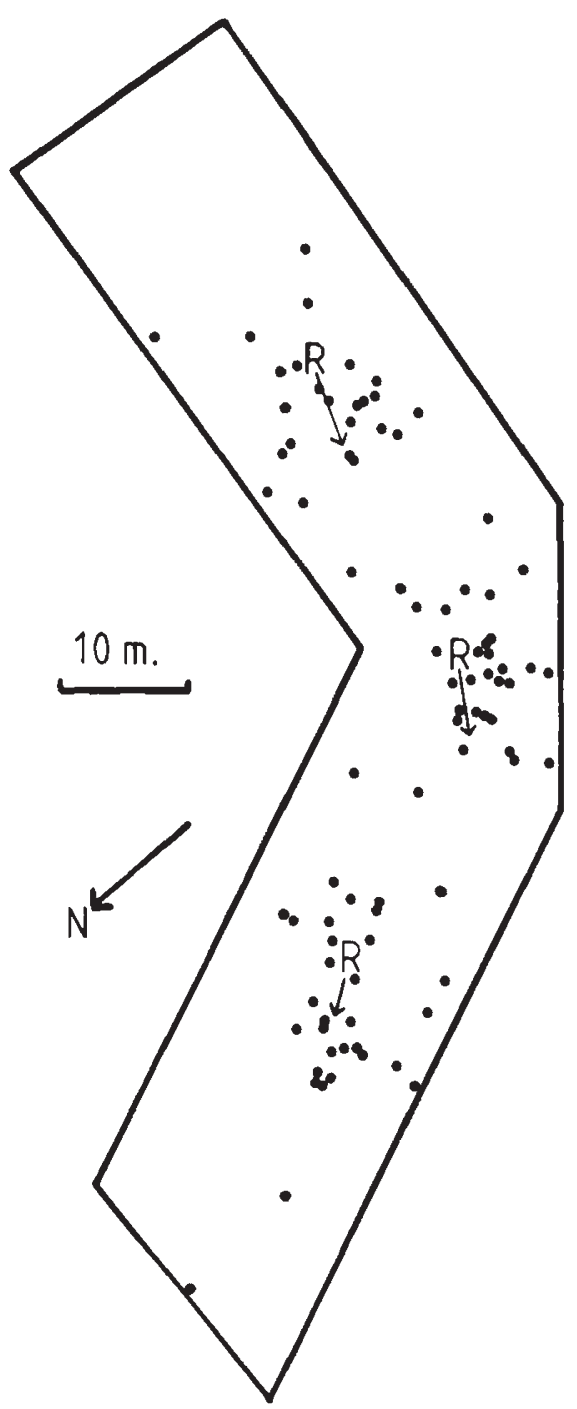

FIG. 1.-Scatter plot of the position of marks after three days dispersal, and the shapes of the three areas searched.

Scorers walked back and forth across the area, recording the position and marks (if any) of grasshoppers seen within a one metre wide strip. The code could usually be read without disturbing the insect, and the numbers seen did not depend on whether the area had been scored already that day. Movements within a day were small compared with those between days, and so only the position of the first sighting on each day has been used.

\section{REsults}

Before looking at the precise position of the marked insects, we will discuss the total numbers seen by each scorer on each of the three days. 
This will give some idea of the consistency of the scoring method, and also estimates of the numbers of marked and unmarked insects within the area. The rate of loss of marked grasshoppers gives information about dispersal, which can be compared with the more detailed spatial data.

\section{(i) Unmarked numbers}

In analysing these data, it has been assumed that the numbers seen follow a Poisson distribution, with a mean which depends on the density of grasshoppers in the area, and the ability of the scorer. Repeated sightings of marked insects by different scorers were independent of each other, but rather more repeats were recorded by individual scorers than expected on a Poisson model. This violation of the statistical assumptions will make the $\chi^{2}$ tests in table 1 too stringent, but since only a minority of the marked

TABLE 1

(a) Analysis of $\chi^{2}$ for unmarked adults

\begin{tabular}{lrrr} 
Effect & \multicolumn{1}{c}{$\chi^{2}$} & df & \\
Scorer & $24 \cdot 05$ & 5 & $* *$ \\
Time & $2 \cdot 30$ & 2 & n.s. \\
Sex $(1: 1)$ & $0 \cdot 22$ & 1 & n.s. \\
Sex $\times$ Scorer & $7 \cdot 37$ & 5 & n.s. \\
Time $\times$ Scorer & $17 \cdot 11$ & 10 & $6 \%$ \\
Sex $\times$ Time & $0 \cdot 54$ & 2 & n.s. \\
Sex $\times$ Time $\times$ Scorer & $5 \cdot 03$ & 10 & n.s. \\
Total & 56.62 & 35 & $*$
\end{tabular}

(b) Analysis of $\chi^{2}$ for nymphs

$\begin{array}{lrrr}\text { Effect } & \chi^{2} & \text { df } & \\ \text { Scorer } & 49 \cdot 65 & 5 & * * \\ \text { Time } & 46 \cdot 65 & 2 & { }^{* *} \\ \text { Time } \times \text { Scorer } & 10 \cdot 45 & 10 & \text { n.s. } \\ \text { Total } & 106 \cdot 75 & 17 & * *\end{array}$

data involved repeated sightings, and the unmarked insects were at a similar density, this should not lead to serious error. The ability of the scorer and the density in each area can be disentangled by maximum likelihood estimation (see Appendix) and it turns out that though scorers do differ in ability, the densities in each area are not significantly different from each other. The "scorer" component can therefore be interpreted unambiguously. Relative abilities are consistent between males, females and nymphs. There is a hint that relative abilities change between days (Time $x$ Scorer), but this is mainly because one scorer had to be replaced after the first day because of illness; the change in ability was neglected in the analysis of $\chi^{2}$.

Turning to more biological features of the data, the observed sex ratio was even on all three days, and the number of adults did not change. However, the number of nymphs fell at about 20 per cent per day, presumably because some died and some became adult. 


\section{(ii) Marked numbers}

The data for the number of marked insects (table 2) can be analysed as for unmarked insects, provided the total numbers seen, including repeated sightings of the same insect, are used. Again, scoring abilities and actual numbers are confounded, but there is no significant difference between areas; this is surprising, since although roughly equal numbers were released in each area, one would have expected the different positions of the release points relative to the edges to affect the rate of loss. As it was, numbers fell substantially over time; the sex ratio remained constant. The relative abilities of scorers differed, but the variation was less pronounced than for unmarked insects $\left(\chi^{2}=15 \cdot 9\right)$. This hints that the paint spots may affect visibility; however, Podisma are brightly coloured anyway, and were easily seen in this open terrain.

TABLE 2

Analysis of $\chi^{2}$ for marked sightings (adults only). The "sex" component is for deviations from the ratio of those released $\left(910^{*}: 80\right.$ )

$\begin{array}{lrrr}\text { Effect } & \chi^{2} & \text { df } & \\ \text { Scorer } & 12.34 & 5 & * \\ \text { Time } & 55.82 & 2 & * * \\ \text { Sex }(91: 80) & 0.98 & 1 & \text { n.s. } \\ \text { Sex } \times \text { Scorer } & 2.50 & 5 & \text { n.s. } \\ \text { Time } \times \text { Scorer } & 11.18 & 10 & \text { n.s. } \\ \text { Sex } \times \text { Time } & 4.19 & 2 & \text { n.s. } \\ \text { Sex } \times \text { Time } \times \text { Scorer } & 4.96 & 10 & \text { n.s. } \\ \text { Total } & 91.97 & 35 & * *\end{array}$

(iii) Estimates of population size

Sightings of individual marks can be used to give an estimate of the numbers present on each day. For simplicity, the differences in scoring ability have been neglected; the design of the experiment ensured that these should average out to some extent. Also, repeated sightings within any one day have not been used; the 171 marks therefore fall into $2^{3}=8$ classes, depending on whether or not they were seen on each of the three days. These data have been fitted to a Poisson model by maximum likelihood (table 3 ). Four parameters were estimated; the chance that a mark would be seen by one or more scorers on a given day, $\theta$, and the proportion remaining in the area $\left(m_{1}, m_{2}, m_{3}\right)$. This method is made more powerful by the assumption that the chance of recapture remains constant. Since care was taken to ensure uniform scoring, and since their relative abilities remained the same from day to day, this assumption seems reasonable.

Table $3 \mathrm{~b}$ shows the estimates for the two sexes separately; the loss in log likelihood when recapture rates are forced to be identical for males and females is $2 \cdot 88$, corresponding to a $\chi_{1}^{2}$ of $5 \cdot 76$. This suggests that females are more easily seen than males; they also seem to be lost faster, though this is not significant. The model fits the data well; $\chi_{4}^{2}=2.85$ after pooling cells with expectations less than 5 . The rapid loss of marks $(\sim 18 \%$ day $^{-1}$ for females, $\sim 12 \%$ day $^{-1}$ for males) could be due either to death or dispersal; this will be discussed later. 
TABLE 3

(a) The observed and expected proportions of insects seen on the various days

\begin{tabular}{|c|c|c|c|c|c|}
\hline \multicolumn{6}{|c|}{ Number seen on } \\
\hline 1st, & 2nd, & 3rd days: & o & 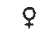 & Expected proportions under the model \\
\hline 0 & 0 & 0 & 39 & 27 & {$\left[1-\theta\left(m_{1}+m_{2}+m_{3}\right)+\theta^{2}\left(m_{2}+2 m_{3}\right)-\theta^{3} m_{3}\right]$} \\
\hline 1 & 0 & 0 & 23 & 26 & $\theta\left[m_{1}-\theta\left(m_{2}+m_{3}\right)+\theta^{2} m_{3}\right]$ \\
\hline 1 & 1 & 0 & 7 & 11 & $\theta^{2}\left(m_{2}-m_{3} \theta\right)$ \\
\hline 1 & 1 & 1 & 5 & 9 & $\theta^{3} m_{3}$ \\
\hline 1 & 0 & 1 & 4 & 4 & $\theta^{2}(1-\theta) m_{3}$ \\
\hline 0 & 1 & 1 & 5 & 1 & $\theta^{2}(1-\theta) m_{3}$ \\
\hline 0 & 1 & 0 & 6 & 2 & $\theta(1-\theta)\left(m_{2}-m_{3} \theta\right)$ \\
\hline 0 & 0 & 1 & 2 & 0 & $\theta(1-\theta)^{2} m_{3}$ \\
\hline & Total & & 91 & 80 & 1 \\
\hline
\end{tabular}

(b) Maximum likelihood estimates for the probability of seeing an insect within the searched area, $(\theta)$, and for the proportion of marked insects remaining after 3,6 and 8 days $\left(m_{1}, m_{2}\right.$ and $\mathrm{m}_{3}$ ). The numbers actually seen are given, for comparison with the estimated numbers present.

\begin{tabular}{|c|c|c|c|c|}
\hline Females & $\begin{array}{l}\theta=0.84 \\
\quad N_{0}=80 \\
\text { (numbers seen }=\end{array}$ & $\begin{array}{c}m_{1}=0 \cdot 73 \\
N_{1}=58 \\
50\end{array}$ & $\begin{aligned} m_{2} & =0 \cdot 39 \\
N_{2} & =31 \\
& 23\end{aligned}$ & $\begin{aligned} & m_{3}=0 \cdot 24 \\
& N_{3}=19 \\
&14)\end{aligned}$ \\
\hline Males & $\begin{array}{c}\theta=0.64 \\
\quad N_{0}=91 \\
\text { (numbers seen }=\end{array}$ & $\begin{aligned} m_{1} & =0 \cdot 74 \\
N_{1} & =67 \\
& 39\end{aligned}$ & $\begin{aligned} m_{2} & =0.45 \\
N_{2} & =41 \\
& 23\end{aligned}$ & $\begin{aligned} & m_{3}=0.39 \\
& N_{3}=36 \\
&16)\end{aligned}$ \\
\hline
\end{tabular}

If the recapture rates for unmarked are equal to those for marked, we can apply the estimate of $\theta$ derived above to the number of sightings of unmarked insects to derive an estimate of the total population size. Over the three days, there were 221 sightings of males, and 231 sightings of females; since there were no significant differences between days (table 1(a)); this gives estimates of 73.7 and 77.0 sightings per day of males and females. Some of these will have been multiple sightings; we can get an estimate of the number of sightings per insect $(\mu)$ from $\theta$, since the chance of $n o t$ seeing an insect is $(1-\theta)=e^{-\mu}$ from the Poisson distribution. Hence, we estimate that there were $\sim 73$ native males and $\sim 42$ females within this $2200 \mathrm{~m}^{2}$ area. The adult population density is therefore $\sim 0.05 \mathrm{~m}^{-2}$.

\section{(iv) Spatial information}

We now come to the main body of the data; the displacements of the marked insects. In all, 169 movements were seen, and the first 89 of these, from release to the first scoring, are shown in fig. 1. Dispersal was directional-the grasshoppers moved W.N.W. regardless of where they started from. It is hard to see why this should be; there is only a shallow slope at this site, and it changes direction between the release points. They may simply have been moving towards a better area; grasshoppers are more abundant just west of the site. It looks as though the displacement ceased after the first three days (fig. 2); this could be because the grasshoppers had then reached a suitable site, or had settled down after their initial disturbance. The observed mean displacement during the first three days was $5.1 \mathrm{~m}$; however, this is less than the true mean, because insects which move further are more likely to be lost. The probability of loss at different 


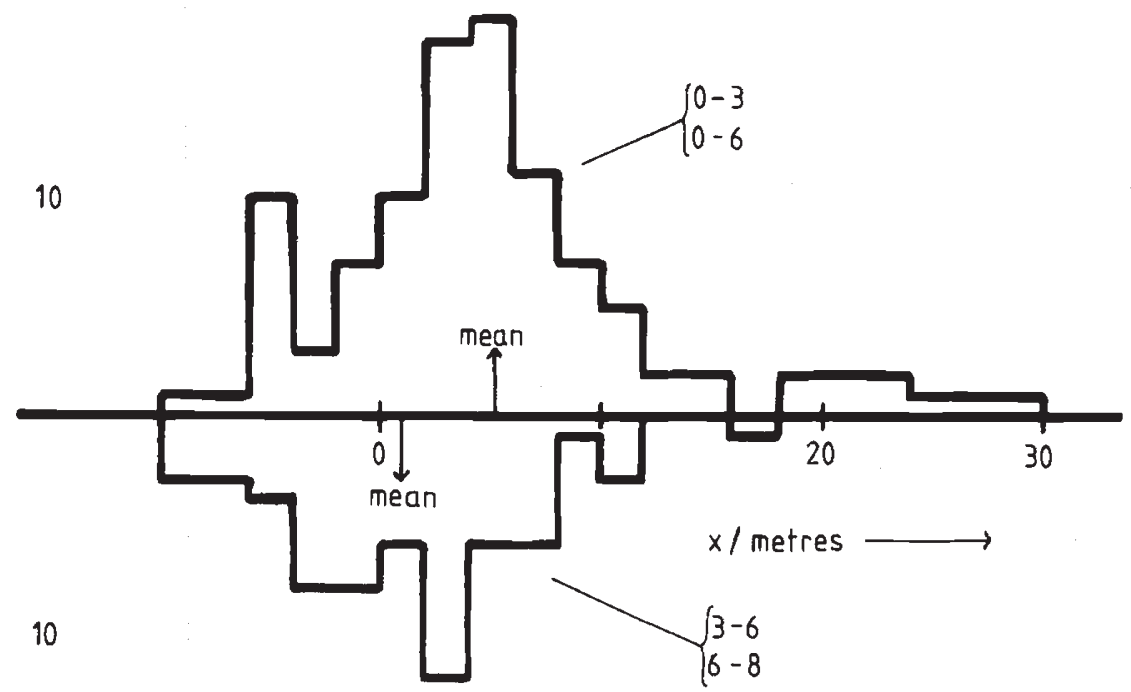

FIG. 2.-Histogram of the displacement along an axis with bearing $310^{\circ}$, over different periods.

distances is determined by the geometry of the searched area (see later), and so we can correct for this bias. In the following, we will assume that the insects moved a certain distance from their release point in the first three days, and then dispersed isotropically about this shifted centre. To estimate the displaced centre, the variance about many possible points was calculated, allowing for loss at large distances, and the point giving minimum variance was chosen; it was ten metres from the release point.

The distribution of distances moved by insects from different release points, and of different sexes, have been compared by using pairwise Kolmogorov-Smirnov tests on the cumulant distributions; no assumptions about the form of the distributions have been made. Despite differences in geometry, no differences were seen between release points. There was no difference between the sexes, despite the faster loss of females noted in the last section.

Both of these tests were restricted to the 89 initial movements, which make up the bulk of the data. After pooling over sexes and release points, the distributions over different time intervals were compared. Fig. 3 shows these after correction by a scale factor $\sqrt{3 / t}$, to allow for a linear increase in variance with time. This correction is only valid if the distribution has been generated by a simple random-walk process. Although no pairwise Kolmogorov-Smirnov test is significant, there is a strong indication that there is more initial dispersal than expected; the dispersal rate over the intervals $0-3$ and $0-6$ was greater than that between 3-6 and 6-8. This may be partly due to a greater loss of long-distance migrants than in later days. The supposition that the distribution expands uniformly with time seems adequate, but there may have been some initial disturbance.

We can thus pool the data from all the observed movements by combining the distributions of fig. 3. However, this observed distribution is biased by the reduced chance of seeing long distance migrants. The probability of seeing a grasshopper at a given radius, $r$, can be calculated by multiplying 


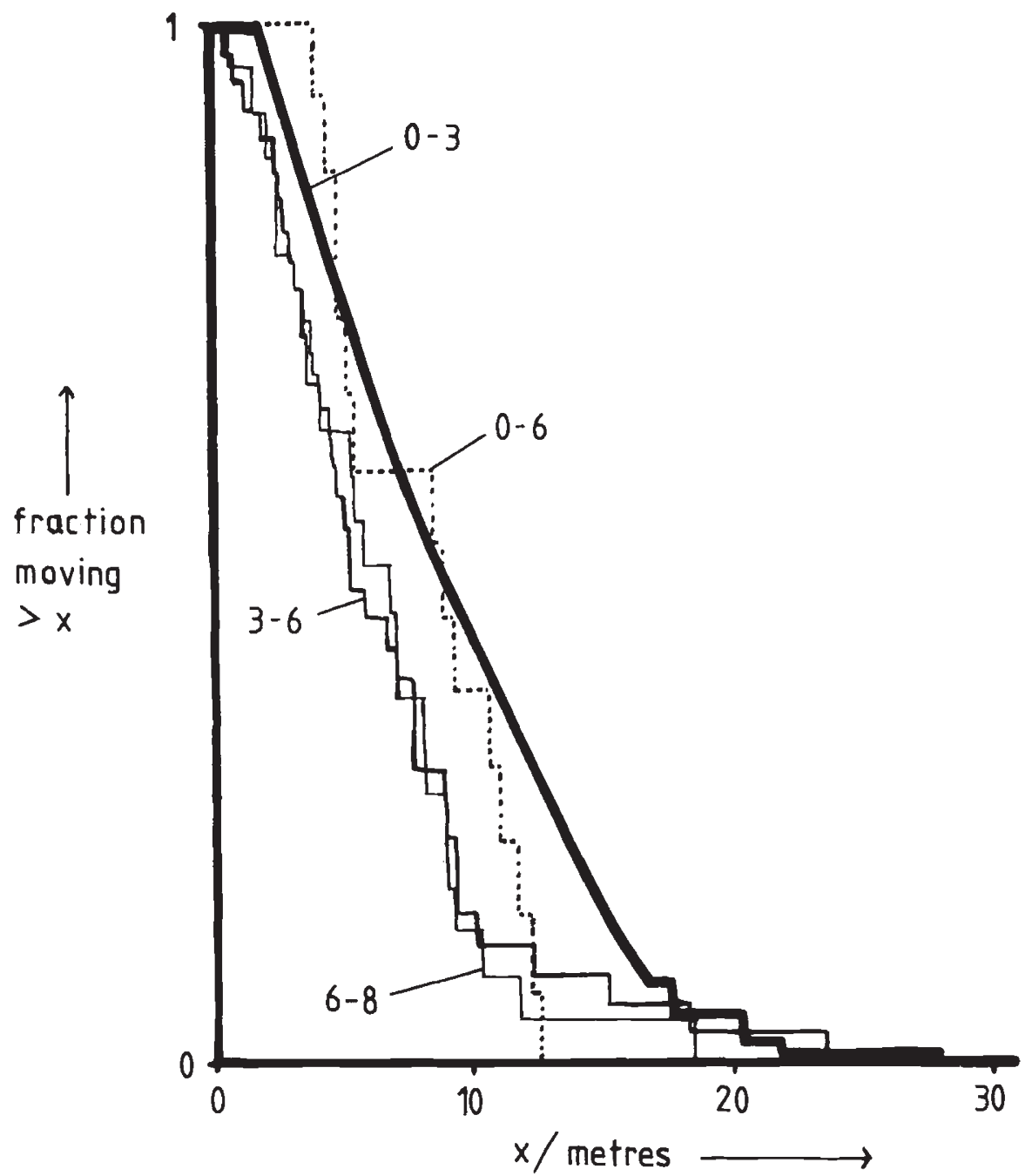

FIG. 3.-Cumulative distribution of distances moved, pooled over sexes and release points. The curves are corrected by a factor $\sqrt{3 / t}$, and so should coincide if the distribution expands uniformly with time.

the chance of seeing an insect in the searched area (estimated as $\theta$ in the last section) by the proportion of the circle with radius $r$ which was searched. This calculation is complicated by the need to combine the distribution of movements over different time periods, and from different starting points. Each starting point has an associated loss rate, and ideally one would use the average of these functions over all starting points. As an approximation, we have assumed that only those insects remaining within the searched area contribute to the average loss rate, and that their loss rate is equal to that for the three (shifted) release points. The numbers remaining within the area are taken from the Poisson model in the last section. 
Using this probability of loss, we have calculated the corrected distribution (fig. 4). It has a radial variance (mean square radius) of $128.4 \mathrm{~m}^{2}$. This estimate is based on only 169 movements, however, and we would like to know how much confidence to put on it; to assess the statistical uncertainties, we must make some assumptions about the form of the true distribution. The estimated distribution is very close to a radial normal curve, as would be expected if all insects move in a random walk. Assuming that it really is normal, we can use a $t$-test to set confidence limits on the variance; at the 95 per cent level, it must lie between $102 \mathrm{~m}^{2}$ and $162 \mathrm{~m}^{2}$. However, this assumption cannot really be justified; most mark-release-recapture experiments show strong leptokurtosis, perhaps because some organisms have a greater tendency to dispersal than others, or because of densitydependent dispersal (see Endler, 1977; Taylor, 1978). It would therefore

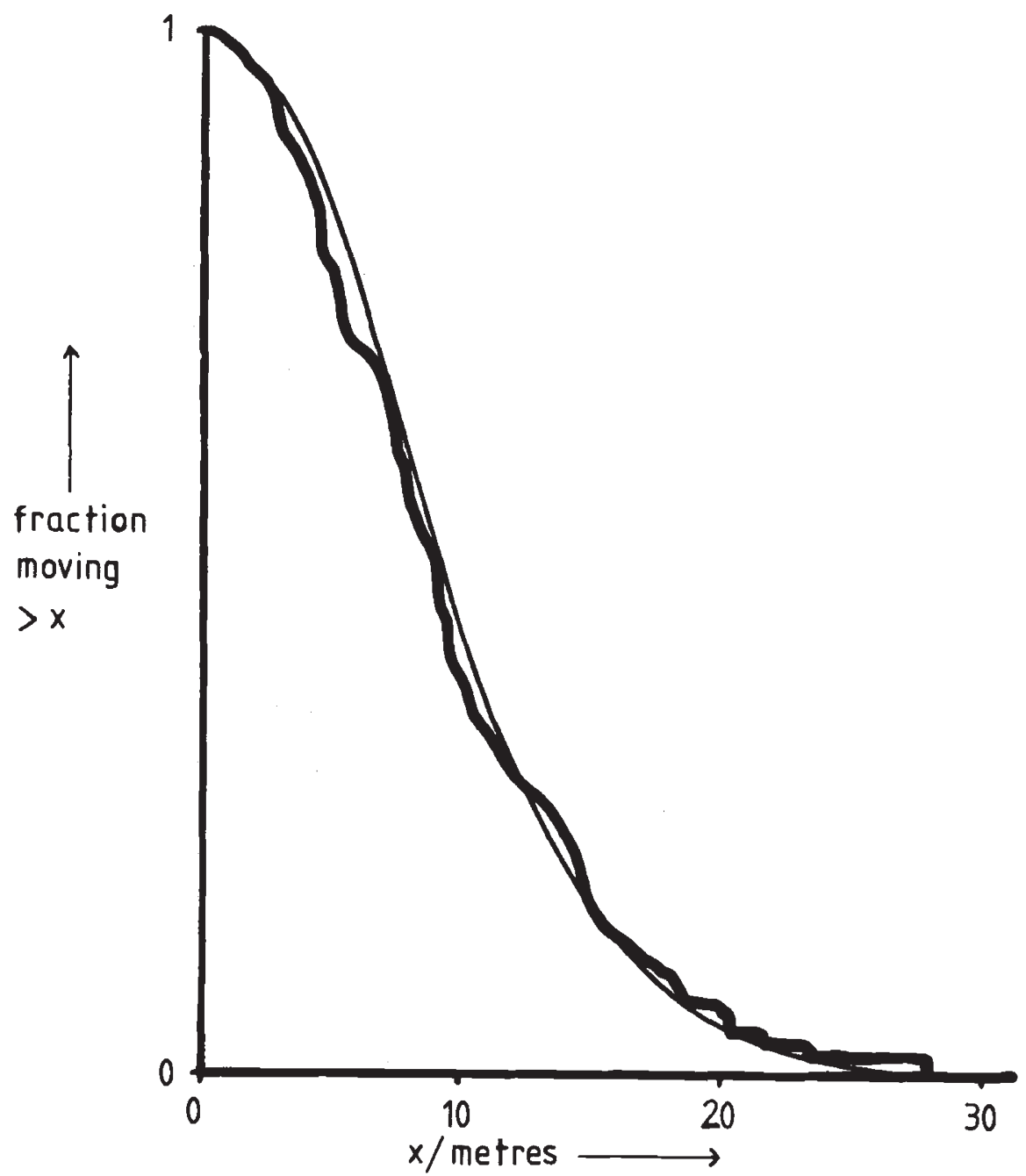

Fig. 4.-Cumulative distribution of all distances moved, corrected by $\sqrt{3 / t}$. The most likely normal curve is also shown. 
be unwise to extrapolate from the body of the distribution, which appears normal, out to the unobserved tails.

If we really can say nothing about the long-distance dispersants, then there need be no upper limit to the variance, and gene flow might be very high; no amount of experiment will resolve this point. However, this conclusion is unduly pessimistic; Podisma is a flightless insect, and probably does not move exceptionally long distances. In any case, a few migrants over distances comparable with the cline width will not matter; they will be swamped by the other type. If we assume that the unobserved migrants do not move further than $40 \mathrm{~m}$, or, equivalently, that the unobserved tail has a mean square at $(34 \mathrm{~m})^{2}$, it is possible to set support limits on the radial variance, subject to the above constraints. As one looks at distributions with a variance further and further from the observed value, their likelihood decreases, and so one can reject variances which lead to a loss in log likelihood of more than 2 units (see Edwards, 1972, for a description of likelihood methods, and Barton, 1979, for details of this application of them). The support limits thus derived are from $104 \mathrm{~m}^{2}$ up to $218 \mathrm{~m}^{2}$; the upper limit depends on the assumption that unobserved migrants move no further than $40 \mathrm{~m}$, but this restriction is unavoidable.

\section{Discussion}

The aim of this experiment was to find the rate of gene flow, or, in the context of relating cline widths to selection pressures, the variance in parent-offspring distance along a given axis. The variance measured during a few days must therefore be extrapolated over the whole life cycle. We will assume that the variance increases linearly with time, as suggested by the near-normal form of the dispersal distribution. It remains to find the mean time spent dispersing between hatching and mating/egg laying. Podisma nymphs are camouflaged, and tend to burrow into the vegetation when disturbed. They are often clumped near to their putative hatching sites. In contrast, the adults (especially the males) are brightly coloured, and try to escape when disturbed. They also move when looking for a mate, or for egg laying sites. We will therefore assume that most dispersal is by adults, and try to find the adult life span.

In principle, the rate of loss of marked grasshoppers could be used to determine mortality (section 3iii). However, much of the loss may be due to dispersal, not death-if the marks spread out in a normal distribution at the observed rate, one would expect losses of 36 per cent, 47 per cent and 50 per cent after three, six and eight days, as they spill over the edges of the searched area. In fact, losses of approximately 27 per cent, 58 per cent and 69 per cent were seen (table 3, pooling sexes). Considering the errors in both sets of figures, the difference signifies little. In any case, the rate of recruitment from nymphs into adults is unknown, since though the numbers of nymphs seen fell, some may have died, and the total number of nymphs cannot be found without assuming that nymphs and adults are equally visible.

We must, therefore, rely on less direct observations of the life history. In the laboratory, females do not begin laying until a week after emergence, and then lay pods of eggs every four or five days. Mating continues throughout adult life. We can thus take ten days as the absolute minimum 
for the dispersal time. In the field, however, largely adult populations may persist for five to eight weeks. This span depends very much on altitude, since populations low down become adult much sooner, and can continue for longer into the autumn. At this particular site, the adult population persists for around seven weeks; allowing a seven day delay before females begin laying, the total fertile life span is $\sim 40$ days. We will therefore work with a mean dispersal time of 20 days.

The marked insects spread into a distribution with variance $128.4 \mathrm{~m}^{2}$ in three days; this variance is the sum of components along two axes, and so the total linear variance in a mean life span of 20 days would be $428 \mathrm{~m}^{2}$, giving an estimate of $\sqrt{m}$, the standard deviation of dispersal, of $20.7 \mathrm{~m} \mathrm{gen}^{-1 / 2}$. A 2-unit support limit of between 18.6 and $27.0 \mathrm{~m} \mathrm{gen}^{-1 / 2}$ can be put around this estimate; however, it only takes account of statistical uncertainties arising from the limited sample size of the experiment. Other errors enter in extrapolating from the observed movements of these particular insects to the general dispersal rate. Movements may have been caused by release and scoring (fig. 3), although some disturbance from cattle and sheep occurs naturally, and there was no evidence of agitation. As discussed above, long-distance dispersal, or long life-spans, might inflate the variance. Most difficult, perhaps, is the inference from dispersal in this open, favourable habitat to the whole area covered by the cline, from a scale of approx. $30 \mathrm{~m}$ up to $\sim 1 \mathrm{~km}$. Ideally one would compare the dispersal rate with the curvature of allele frequency at the same site, but frequency differences cannot be measured over such a fine scale. Although Podisma is fairly abundant throughout this region, it is still patchily distributed, and it is not clear how this patchiness affects dispersal. In an unfavourable habitat, dispersal might be greater (witness the initial shift west?), but on the other hand, grasshoppers would be reluctant to leave suitable sites. On the whole, we think it likely that the dispersal rate could be slightly lower than the statistical lower bound of $18.6 \mathrm{~m} \mathrm{gen}^{-1 / 2}$.

Despite the many possible errors, this estimate of dispersal $(\sqrt{m}=$ $20.6 \mathrm{~m} \mathrm{gen}^{-1 / 2}$ ) is still useful. It is certainly much lower than the width of the clines in karyotype $(800 \mathrm{~m})$ and hybrid inviability $(350 \mathrm{~m})$, and hence the selection on each locus involved in these differences must be weak (approx. 0.5 per cent and 3 per cent respectively; Barton and Hewitt, $1981 a, b)$. The dispersal rate forms the one link between spatial scales and selection strengths.

How can the estimate be improved? The primary difficulty is not the size of the mark-release-recapture experiment, but the extension of its results to the complete generation length. One could measure gene flow directly by releasing organisms containing marked genes (as in Dobzhansky and Wright, 1947). The only easy marker in Podisma is the chromosome difference itself, and transplants have been made between populations on either side of the chromosomal cline (Barton, 1980). However, only seven foreign grasshoppers were recovered, probably because hybrids had reduced viability. These seven had a linear variance of only $50 \mathrm{~m}^{2}$ about the release point after two years, suggesting that the direct estimate described here may be too high. The transplant site was bounded by water on two sides, though, and so it is hard to compare the figures. In general, although transplants give direct measures of gene flow, they are laborious, and still cannot give information for a large area. 
Another way of estimating gene flow directly is to look at the typical width of random deviations in allele frequency. Populations cannot differentiate over scales smaller than the dispersal distance unless selection is intense. This argument has been used qualitatively to set an upper limit on dispersal (e.g., Taylor and Powell, 1977), and has been applied quantitatively to lethal allelism rates in Drosophila by Yokoyama (1979). In Podisma, populations less than $100 \mathrm{~m}$ apart can still show significant differences in karyotype frequencies (Barton and Hewitt, 1981a), and a more detailed study now in progress should give a better idea of local dispersal. The method is limited by the number of close pairs of populations which are collected, but can give average estimates over a larger region than can physical or genetic marking.

This analysis of local differentiation will also give an estimate of the neighbourhood size needed to produce the fluctuations. Neighbourhood size, defined by Wright (1951), is a measure of the strength of sampling drift, and is the effective number of individuals within a dispersal range $\left(N_{e}=4 \pi \rho_{e} m\right)$. The current data on the chromosomal cline give a rough estimate of $N=27$ (Barton and Hewitt, 1981a). The estimates presented here of adult population density $\left(\rho=0.05 \mathrm{~m}^{-2}\right)$ and dispersal range ( $m=$ $428 \mathrm{~m}^{2}$ ) lead to a neighbourhood size $N=300$. The discrepancy seems large, but the direct estimates refer only to one small part of the cline, which probably has a higher density, and perhaps higher dispersal, than average. It also refers to the actual population density, which may be much greater than the effective density (see Wright, 1978). It is hard to relate direct observations to the underlying genetic parameters, a difficulty especially apparent in studying population structure.

In conclusion, we should note that this paper has only been concerned with one type of dispersal-the gradual diffusion of alleles between neighbouring areas, over time scales of a few generations. Over long periods, this type of flow may be negligible in comparison with extinction and recolonisation processes. For example, the Alpes Maritimes were covered by ice during the last glaciation, less than 10,000 years ago, and the expansion of the southern karyotype into its present distribution probably had little relation to the slow diffusion within populated regions observed here. As well as applying mainly to short timescales, diffusive flow may be irrelevant to the spread of allelic substitutions which are incompatible with their ancestral form; these may spread by extinction and recolonisation of demes (Lande, 1979), a process almost impossible to study directly.

Acknowledgements. - We are grateful for the help in the field of Bruce and Helen Halliday, James, Matthew, Daniel and Elizabeth Hewitt, and for the hospitality of M et Mme Aviotti. This work was supported by an N.E.R.C. Studentship to the first author, and an S.R.C. research grant to the second.

\section{REFERENCES}

BARTON, N. H. 1979. A Narrow Hybrid Zone in the Alpine Grasshopper Podisma pedestris. Ph.D. Thesis, University of East Anglia, Norwich.

BARTON, N. H. 1980. The fitness of hybrids between two chromosomal races of the grasshopper Podisma pedestris. Heredity, 45, 47-59.

BARTON, N. H., AND HEWITT, G. M. 1981a. A chromosomal cline in the grasshopper Podisma pedestris. Evolution, 35, 1008-1018. 
BARTON, N. H., AND HEWITT, G. M. 1981b. The genetic basis of hybrid inviability between two chromosomal races of the grasshopper Podisma pedestris. Heredity, 47, 367-383.

DOBZHANSKY, TH., AND WRIGHT, s. 1947. Genetics of natural populations: XV. Rate of diffusion of a mutant gene through a population of Drosophila pseudoobscura. Genetics, 32, 303-324.

EDWARDS, A. W. F. 1972. Likelihood. Cambridge Univ. Press.

ENDLeR, J. A. 1977. Geographic Variation, Speciation and Clines. Princeton Univ. Press.

HALDANE. J. B. S. 1948. The theory of a cline. J. Genet., 48, 277-284.

LANDE, R. 1979. Effective deme sizes during long-term evolution estimated from rates of chromosomal rearrangement. Evolution, 33, 234-251.

NAGYLAKI, T. 1975. Conditions for the existence of clines. Genetics, 80, 585-615.

TAYLOR, C. E., AND POWELL, J. R. 1977. Microgeographic differentiation of chromosomal and enzyme polymorphisms in Drosophila persimilis. Genetics, 85, 681-695.

TAYLOR, R. A. J. 1978. The relationship between density and distance of dispersing insects. Ecol. Entom., 3, 63-70.

WRIGHT, s. 1951. The genetical structure of populations. Ann. Eugenics, 15, 323-354.

WRIGHT, S. 1978. Evolution and the Genetics of Population. Vol. IV. Variability Within and Among Natural Populations. Chapter 2. Univ, of Chicago Press.

YOKOYAMA, S. 1979. The rate of allelism of lethal genes in a geographically structured population. Genetics, 93, 245-262.

\section{APPENDIX \\ Estimation of partially confounded components}

The number of insects seen $(n)$ depends on the ability of the scorer $(i)$, the density actually present in the area $(j)$, and the day of the observation $(k)$. If sightings are independent of each other, $n$ will follow a Poisson distribution with mean $\mu$ :

$$
\operatorname{Prob}(n)=\frac{e^{-\mu} \mu^{n}}{n !} \text {. }
$$

If we also assume that the various effects do not interact, then $\mu$ can be written as:

$$
\mu=S_{i} A_{j} T_{k} \text {. }
$$

The log likelihood for the set of observations, $\{n\}$, is therefore given by:

$$
L=\sum\left[n \log \left(S_{i} A_{j} T_{k}\right)-S_{i} A_{i} T_{k}\right]+\text { constant. }
$$

To derive maximum likelihood estimates of $S_{i}, A_{j}$ and $T_{k}$, we differentiate with reference to each of the three parameters, and set the differential to zero:

$$
0=\frac{\sum n}{\hat{S}_{i}}-\sum \hat{A}_{i} \hat{T}_{k},
$$

where the sum is taken over all cells involving scorer $i$.

$$
0=\frac{\sum n}{\hat{A}_{j}}-\sum \hat{S}_{i} \hat{T}_{k},
$$

where the sum is taken over all cells involving area $j$

$$
0=\frac{\sum n}{\hat{T}_{k}}-\sum \hat{S}_{i} \hat{A}_{i}
$$

where the sum is taken over all cells involving time $k$.

These equations can be solved iteratively to give $\dot{S}_{i}, \hat{A}_{i}, \hat{T}_{k}$. 